\title{
LA PRESENCIA DEL POSTMODERNISMO EN EL DEBATE FILOSÓFICO NÓRDICO
}

\author{
Morten WALLENTINSEN
}

El supuesto de que la postmodernidad sólo haya sido asunto de la filosofía francesa, americana e italiana fundamentalmente cae por los suelos al investigar lo que se debate en, por ejemplo, la filosofía nórdica. Desde muy pronto, a principios de los noventa del siglo pasado, los intelectuales de Dinamarca, Noruega y Suecia leían al filósofo alemán que luego marcará época con sus obras, Friedrich Nietzsche, aunque «no se puede aprender nada sorprendente sobre Nietzsche de Brandes, Hansson, Garbor, Strindberg o Ibsen" porque "todos ellos le leyeron a partir de sus propias necesidades" (Trond Berg Eriksen, Nietzsche og det moderne, Universistetsforlaget, Oslo, 1989, p.29). A parte de la lectura de Nietzsche, algunos de ellos, Brandes y Strindberg, tenían correspondencia con el mismo y el filósofo alemán llegó a leer a Strindberg (íbid., pp. 30-1). No obstante, esta generación de literarios introdujo la crítica de la ciega creencia en el progreso, de la modernidad y de la racionalidad misma. Con la publicación de la obra maestra de Knut Hamsun, Sult [hambre] (1890), ya se había indicado el alcance de la crisis de la racionalidad. Tampoco puede olvidarse que el país de origen de Kierkegaard es Dinamarca. En efecto, ellos marcaron de distintas maneras lo que iba a ser un tema filosófico importante. Además, la estrecha relación con la filosofía anglosajona también condujo a que el postmodernismo estuviera presente desde muy temprano (Dagfinn Føllesdal, Jaakko Hintikka y Georg Henrik von Wright). Asimismo, la filosofía wittgensteiniana formó parte de la discusión filosófica de los países del norte y junto con ella vinieron los demás representantes de lo que podríamos llamar el postmodernismo.

Este artículo no tiene el objetivo de investigar estas relaciones de manera exhaustiva sino que, por el contrario, trata de tomar una obra particular como 
base de esta tesis y mostrar cómo Øyvind Pålshaugen, Kritikk av den ene fornuft [Crítica de la única razon] Adorno, Derrida y Wittgenstein contra Habermas, Oslo: Spartacus Forlag, 1997, se sitúa en este debate. El autor noruego es Dr. Philos. que encabeza la investigación en el Instituto de trabajo de Noruega.

A través de un viaje crítico, el autor Øyvind Pålshaugen nos acerca a tres personajes filosoficos muy importantes, Adorno, Derrida y Wittgenstein, criticados por otro no menos importante, Habermas. La introducción empieza con el tírulo: "La Responsabilidad de los intelectuales" mostrando tres aspectos del giro lingüístico, a saber, «el retroceso de la ilustración: ¿un mito?», "¿manejamos el arte de leer y escribir?" y "ihay una ciencia postmoderna?». La responsabilidad pertenece a Habermas y los aspectos del giro lingǘstico reflejan los trabajos, respectivamente, de Adorno, Derrida y Wittgenstein.

En el debate público cada vez participan menos intelectuales porque el público ha perdido fe en ellos y los intelectuales han perdido fe en el debate público porque cada vez se parece más a Show Biz. Aquí empieza el problema (Pålshaugen 1997:8):

"Si sólo una minoría de las cuestiones fundamentales se pueden discutir bajo la dirección del experto, y la sabiduría profesional así vista, resulta siempre insuficiente en los casos importantes, entonces es necesario una crítica por parte de los intelectuales dentro de la profesión».

Por lo tanto se trata de una cuestión interna. Este tipo de crítica se manifestó abiertamente durante los años sesenta con la critica al positivismo; en los setenta, la crítica feminista; y durante los ochenta, la crítica filosófica a la razón. Estas corrientes estaban de moda entre los intelectuales de aquellas épocas (Pålshaugen 1997:9). Ahora bien, lo que sí estaba claro desde el principio, era que los intelectuales eran participantes activos, no sólo observadores, de los fenómenos sociales y culturales que fueron objeto de investigación. Aún más interno es el problema del lenguaje, debido al giro lingüístico (Rorty, 1967). Por este giro la razón ha obtenido un oponente interno. Cuando la razón y por tanto la mismísima instancia a que el intelectual apela se cuestionan, el intelectual tiene que entregarse a la ironía, el humor, la retórica o callarse para lograr el dominio del argumento (Pålshaugen 1997:11). El debate es, por así decirlo, un juego de palabras. Una vez expuesto el problema, se dirige a Habermas cuyo proyecto ha sido la cuestión de la unidad de la razón. A través de un análisis filosófico del lenguaje, Habermas ha intentado demostrar que la 
posibilidad de la racionalidad está en nuestra comunidad como usuarios del lenguaje; es la unidad formal de la razón. Por eso, Habermas está en constante polémica con los llamados relativistas. Veamos, pues, cómo Habermas entiende las posiciones relativistas y luego cómo, de modo categórico, las hace callar (Pålshaugen 1997:14-5):

"Los relativistas, o los contexualistas, afirman, según Habermas, que cada descripción posible sólo refleja una construcción particular de la realidad, y que no hay ningún estándar de la racionalidad que vaya más allá de las implicaciones de la cultura local [el contexto]. Tal hipótesis relativista... no se puede practicar sin suspender las reglas del juego, es decir, que el contenido de la frase es contradictorio a la acción lingüística; una contradicción ejecutiva".

Éste es el error que comete el relativista que quiere ir más allá de su contexto con la acción lingǘrtica; la acción de comunicarse contradice el contenido, que a su vez dice que no puede comunicarse fuera del contexto. Sin embargo, aún queda Rorty, que es más listo que éstos, y que afirma que ula verdad" no se refiere a un valor propio de la verdad. El valor de la verdad lo da la cultura en que está implícito y, por esta razón, el enunciado es inteligible al apelar al valor de la verdad. Más allá no quiere ir Rorty, según Habermas, y es por lo tanto un participante. Así Habermas hace callar también a Rorty, un representante de la racionalidad particular.

Ahora bien, el autor termina la introducción preguntando qué podría hacer común a la razón. Este es el punto de partida para el análisis del libro. Los tres ensayos están escritos para demostrar cómo Habermas llama a tres voces muy distintas intentando acallarlas. Hay tres elementos de los ensayos que podrían justificar la forma de un libro, es decir, que los dota de unidad:

1) enseñan como Habermas los hace representantes de la racionalidad de manera particular y de este modo tienen que callarse ante él,

2) van descubriendo diferencias con la lectura de Habermas y lo que dicen los tres mencionados autores, $y$

1 Sus esfuerzos por construir una teoría normativa con un nuevo concepto de la verdad los reúne en Theorie des kommunikativen Handelns I und II, Frankfurt a. M.: Suhrkamp Verlag, 1981. 
3) reúnen lo común de los tres filósofos - las preguntas críticas sin respuestas.

Por lo tanto el autor concluye negativamente ante el proyecto de Habermas y afirma que la racionalidad tiene que ser construida en cada caso; el intelectual siempre será entonces un sujeto constitutivo del argumento. Vayamos, pues, a ver un resumen de cada uno de los ensayos, empezando con Adorno.

I. La modernización de la teoría crítica de Habermas: El retroceso de la ilustración, ¿un mito?

Adorno y Habermas están de acuerdo en que la ciencia es necesariamente subjetiva y en que la subjetividad no impide que haya una ciencia. Sin embargo, Habermas, al justificar la ciencia normativa, se aleja substancialmente de Adorno. Este propósito no es una continuación de la teoría crítica, pero tampoco carece de toda conexión ${ }^{1}$. Habermas es consciente de que ha sido Adorno, dentro la teoría crítica, el que ha reflexionado sobre el problema dialéctico de ser participante y al mismo tiempo investigador. Ante la posición de Adorno, Habermas formula su crítica al problema. El objetivo de la crítica es aprender de las aporías de Adorno y a través de ellas justificar un cambio de paradigma para resolver los problemas anteriores (Pålshaugen 1997:21).

Los antecedentes de la obra de Adorno y Horkheimer ${ }^{2}$ son el marxismo y las interpretaciones que hicieron de Weber y Lukács. Sin entrar en esta extensa literatura, expone Pålshaugen (1997:22-3) que el horizonte marxista no les bastó para explicar cómo el capitalismo logró aumentar la capacidad productiva y al mismo tiempo frenó la resistencia subjetiva. El concepto de cosificación, de Lukács ${ }^{3}$, tiene un límite que según Habermas no podrían aceptar Adorno y Horkheimer: empíricamente, por la cosificación extensa con referencia a la industria cultural que ha cosificado el interior del hombre; teórica-

2 La lectura fundamental para la comprensión de esta parte es la Dialektik der Aufklärung por HORKHEIMER y ADORNO (Frankfurt a. M.: Fischer Verlag, 1969).

3 Al sustituir la racionalización de Weber por el concepto de cosificación de Marx, intenta poner de relieve que las tendencias de cosificación que aparecen como naturales están conectadas con el capitalismo y se pueden abolir con él. Sin embargo, no piensa en que se podría llegar a una cosificación total del sujeto y por lo tanto pone fe en que la clase trabajadora, como sujeto, puede acabar con el capitalismo. 
mente, no podrían aceptar la tesis de que la subjetividad no se puede objetivar completamente porque representa un pensamiento que deja que los conceptos sean más reales que la realidad misma. Hay que radicalizar la crítica, y por eso el problema de Adorno y Horkheimer era que necesitaban un aparato de conceptos que pudiera hacer posible rechazar la totalidad como falsa ${ }^{4}$. Estos conceptos tienen, sin embargo, la misma ambición que los sistemas que ellos critican. ¿Cómo logran este objetivo y a qué precio? es la pregunta de Habermas. Hay tres elementos clave en el análisis (Pålshaugen 1997:23-4):

1) la "razón subjetiva" y el "pensar identificativo" son conceptos fundamentales y no derivados, que cobran valor universal por el capitalismo,

2) la Zweckrationalität es la causa de la cosificación; el hombre se reproduce trabajando, $y$

3) esta racionalidad instrumental no sólo cosifica la naturaleza externa sino también la interna. Se objetiva su propia naturaleza.

La crítica a la racionalidad instrumental deja ver que es una racionalidad pervertida - es un instrumento para la represión de la naturaleza propia de los sujetos. La tarea del pensamiento crítico es reconocer la represión que llega hasta dentro del pensar. Pero, pregunta Habermas, ¿cómo podrá este pensar salir de la represión? Horkheimer y Adorno mantienen aún que pensar es un pensar identificativo. Con conceptos se manipula la realidad. El pensar, entonces, no tiene sentido fuera del ámbito de la racionalidad instrumental. La respuesta de Habermas es que hay que evitar la dialéctica positiva de Hegel, el sistema, sin que se deje de filosofar. La respuesta es, por lo tanto, una dialéctica negativa y no acabada, sino abierta. El precio que paga Adorno, según Habermas, es que deja a un lado la ambición de hacer una teoría crítica.

Ahora bien, leer la obra de Horkheimer y Adorno da lugar a otra interpretación - la de que son conscientes del problema interno de una racionalidad instrumental crítica. El hilo conductor no sería ya el estado de los conceptos sino sus usos (Pålshaugen 1997:27). La reflexión sobre la barbarie moderna estaba ya en el pensamiento ilustrado. La tesis no es que el interés esté fuera del pensamiento sino que está en el pensamiento mismo, no viene impuesto desde fuera. Progresión y retroceso son posibilidades de la ilustración a la vez.

4 Véase Theorie, 1981, I:506, „...das Ganze als das Unwahre zu denunzieren». 
Se ve entonces que no mantienen que la racionalidad instrumental se apropie totalmente del pensamiento aunque es la tendencia actual. El problema, para ellos, es cómo reconocer la cosificación del pensamiento y cómo luchar contra ella. La respuesta es, por vía de la reflexión crítica, dejar ver la construcción histórica de los usos de los conceptos (Pålshaugen 1997:29). En esta reflexión hay una dialéctica; el sujeto pensante está subordinado a la lógica del entendimiento y a la vez éste la maneja libremente en el intento. La ilustración es la transición del Mythos al Logos en la que no sólo se conocen las leyes naturales sino que cambia también el cómo entenderse como hombre. La Dialektik der Aufklärung se resume así: "el éxito del pensamiento de la ilustración, la progresión ante los mitos, ha llevado a la creencia de que con esta lógica está el camino principalmente abierto para que el sujeto entienda completamente su mundo a través de los conceptos" (íbid.). Adorno y Horkheimer critican la identidad entre concepto y realidad. Apuntan a la situación histórico-natural del lenguaje, que no se puede abolir; el concepto siempre va más allá que su objeto y el objeto (Ding an sich) también lo hace. Atacan, por lo tanto, la identidad. Con esta reflexión histórico-natural se puede mantener el camino abierto hacia la verdad recordando que no es la verdad absoluta (de Hegel). El punto clave de esta crítica es dejar que abra el camino para experiencias que van más allá de los conceptos.

La naturaleza del sujeto no es un concepto positivo tal como lo analiza Habermas sino que ésta se utiliza como concepto límite de nuestro conocimiento. La emancipación consiste en reconocer los límites del pensamiento natural-histórico, el no ser esclavo del concepto positivo (Pålshaugen 1997:33). Esta libertad se entiende dialécticamente; el sujeto está libre en cuanto consciente de su relación con el lenguaje y no lo está como usuario de este lenguaje (Pålshaugen 1997:34). La teoría crítica de Adorno es exactamente eso, poner de relieve la dialéctica histórico-natural. A través del desplazamiento de esta dialéctica la ilustración perdió la posibilidad de crítica interna. Se ha prohibido de esta manera pensar lo que no se puede pensar fuera de la lógica. La lógica discursiva, a la que el sujeto está subordinado, queda más abierta para la experiencia por la crítica que hace Adorno y no es, como interpreta Habermas, una crítica al uso de los conceptos per se porque sólo con conceptos pueden lograr lo que los conceptos limitan (Adorno, Negative Dialektik, Frankfurt a. M.: Suhrkamp Verlag, 1973:62).

La exigencia de mantener la naturaleza del sujeto no es un criterio positivo en el sentido científico, y justamente eso da lugar a una dialéctica (personal) 
entre teoría y práctica. Esta consecuencia es la que no ve Habermas; pone los conceptos contra la exigencia de la lógica discursiva, pues, leer a Adorno de esta manera justifica el punto de vista de Habermas y limita el alcance de la crítica. Su lectura está, por lo tanto, condicionada por la lógica discursiva, que reconoce como propia del pensamiento mismo (Pålshaugen 1997:36).

Habermas y Adorno coinciden en que la teoría social tiene que ser normativa. Habermas se esfuerza, a través de su obra, en justificar su posición, su teoría normativa, mientras para Adorno era importante demostrar el carácter subjetivo, es decir, histórico-natural, de la lógica discursiva que aparentemente parece objetiva. La subjetividad teórica tiene que tratarse como condición para toda la teoría. La lógica se construye históricamente y se la mantiene a través del uso (natural). El concepto de la lógica natural es una manera para el sujeto de darse cuenta de su límite sin que este límite pueda ser conceptualizado de manera positiva. La lógica aparece entonces como una transmisión entre la subjetividad - el aspecto histórico - y la naturaleza — su necesidad. Así siempre se presupone la dimensión normativa.

Pålshaugen concluye el ensayo afirmando que la filosofía de Adorno mantiene una actualidad "a pesar de los esfuerzos de Habermas para enterrarla en el mausoleo de la historia de la teoría» (Pålshaugen 1997:39). La actualidad consiste en su perspectiva de la verdad y cómo el conocimiento verdadero de fenómenos sociales es posible sin una teoría total. Mantiene que hay que renunciar al proyecto de totalizar la realidad pero sin que se tenga que renunciar a tener en cuenta la totalidad.

\section{II. ¿Manejamos el arte de leer y escribir? Comentarios a la lectura que hace Habermas de Derrida}

El giro lingüístico en las ciencias sociales había pasado inadvertido, antes de que Habermas lo tomara en serio. En Sociologla esto ha creado, de manera radical, un problema interno. Habermas mantiene que la filosoffa actual se puede caracterizar por la transición desde la filosofía de la consciencia al nuevo paradigma lingüístico. La Teoría de la acción comunicativa introduce fundamentalmente el giro lingüístico en el ámbito de las ciencias sociales. El paradigma lingǘstico domina ya el debate, pero aún no se ha cristalizado en un resultado concreto. En Der philosophische Diskurs der Moderne (Frankfurt a. M.: Suhrkamp Verlag, 1985) Habermas lee a Derrida (entre otros) que es obje- 
to de este ensayo. Según Habermas, Derrida va demasiado lejos renunciando a la distinción entre la retórica y la lógica.

De un plumazo pone a Derrida en la misma categoría que a Adorno por su fuente común más o menos explícita: Nietzsche. El mantener la subjetividad contextual no les ha impedido escribir obras extensas - caen en la falacia de las expresiones ejecutivas (performative utterances). A través de la retórica, Nietzsche hace patente que el problema de estilo lleva implicaciones para el conocimiento. En esta experiencia del lenguaje y su forma tienen Nietzsche, Adorno y Derrida algo en común, eso sí. Sin embargo, en su tratamiento se distancian necesariamente porque "la respuesta tiene que ser personal" (Pålshaugen 1997:53).

Para Derrida, el leer los textos con cuidado es su estrategia filosófica porque «el lenguaje sólo se puede problematizar a través del uso del lenguaje» (f́bid.), por ejemplo, en textos concretos. El método de desconstrucción se basa internamente en estos textos. La elección de textos cobra importancia así en su filosofía, sin embargo, no existe $E l$ texto. Los textos dependen de su contexto, es decir, siempre hay que preguntar en qué discurso estamos y quién quiere intervenir (Cf. Kierkegaard). Por eso es difícil criticar a Derrida sin tener en cuenta estos dos aspectos. Por esto, la crítica de Habermas da la impresión de carecer de sentido bien por los textos que elige o bien por no fundamentar su crítica en los textos mismos de Derrida (Pålshaugen 1997:54).

Aquí hallamos mi primera objeción al libro. El autor cita la obra de Derrida y con gran sorpresa aparecen estas palabras: «Signature Event Context». Naturalmente, el autor está limitado, como cualquiera, por no saber francés, ya que el texto citado está publicado originalmente en francés en Marges. El autor tiene que mantener que la traducción de una lengua a otra sí es posible ${ }^{5}$ porque lee a Derrida en inglés en lugar de francés. Sin embargo, al mantener el giro lingüístico, comete, por así decirlo, la falacia de las expresiones ejecutivas.

Teniendo en cuenta esta posible falacia vayamos al texto. En cuanto a la comunicación, tema del texto, Derrida distingue entre "dissémination y «com-

5 Antonio Bolfvar BotiA en, El Estructuralismo: De Levi-Strauss a Derrida, Madrid: Editorial Cincel, 1985:177, afirma básicamente lo contrario sobre los términos del filósofo francés, y debemos tenerlo en cuenta desde la perspectiva de una lengua que está más cerca del francés que del inglés. 
munication como dos perspectivas distintas del lenguaje. La primera expresa la idea de que la semántica no se transmite fácilmente sin que haya alteraciones, ya que nuevos sentidos pueden aparecer a través de la transmisión. La otra perspectiva no deja espacio para estas alteraciones. Por vía de l'écriture le permite un espacio desde el que estudia el lenguaje como medio de comunicación. Su punto de partida es el filósofo francés Condillac que afirmó que la escritura es mimesis. Derrida llega a la misma conclusión desconstruyendo el lenguaje en tres etapas, a saber, lenguaje mediante geste, voix, écriture. Ahora bien, después de esta interpretación, investiga los tres aspectos más importantes del lenguaje: absence, règle y présence. El hilo conductor es que la escritura representa una idea mental que es un intento de representar un objeto concreto. La escritura se condiciona por la "ausencia" de aquello de que se habla y así permite la comunicación. Además de la ausencia de las imágenes mentales y los objetos concretos también brilla por su ausencia el autor. Este absence implica más que la no-presencia o distancia del autor, que el lenguaje necesite una determinada estructura, la regla es poderse repetir siempre. La repetición implica que haya identificación entre lo que escribió el autor ausente y lo que lee el lector presente (inmanencia de sentido). Sólo entonces se puede decir que el signo crea significado, de modo que se entiende que la presencia y la ausencia no son conceptos simplemente contrarios sino que una vez disueltos como conceptos dialécticos el nuevo sentido del análisis desplaza su relación interna. Además de alterar la relación entre ambos conceptos, se desplaza el discurso del que son parte; se abre un espacio para un conocimiento nuevo.

Este análisis se repite en cuanto a la parole llegando a la sorprendente conclusión de que no hay diferencia entre la estructura del habla y la escritura; su lógica es la estructura grafemática, y lo que es más: "cada expresión lingüistica, oral o escrita, tiene una dimensión material, de naturaleza fonética o grafémica, que va más allá, que es distinta del sentido de la expresión que se puede caracterizar como la dimensión ideal" (Pålshaugen 1997:61). La diferencia entre ambos es absoluta. Sin embargo, al crear sentido en el juego de diferencias y ausencias no se pueden tomar las dimensiones puramente ideales o materiales. En otras palabras, más allá de cualquier creación de sentido, en cada expresión existe ya un elemento en un juego donde lo inteligible y lo sensible actúan. Las diferencias fonéticas y grafemáticas no tienen en sí sentido, pero en el juego crean los sentidos, es decir que las reglas que el sujeto sigue no son sólo de naturaleza gramatical o lógica sino que hay otro orden de reglas, el poder identificar la expresión fonética o escrita. 
Volvamos, pues, a ver cómo Habermas lee a Derrida. Dice: «Derrida quiere extender la soberanía de la retórica en el dominio de la lógica para solucionar el problema que una crítica de la razón total supone" (Habermas 1985:221) ${ }^{6}$. Por no estipular su propia posición, Habermas piensa que acaba en una contradicción que consiste en que Derrida no puede argumentar para la posición desde la que critica a las otras posiciones en cuanto a la racionalidad. Cuando la lógica pierde su autoridad ante la retórica, contradicción e inconsistencia son las consecuencias.

El objetivo de Habermas, leyendo a Derrida, es crear claridad. Por eso no se entiende muy bien el porqué Habermas deja al lado los textos de Derrida y comienza una lectura de J. Cullers, un comentarista de Derrida, diciendo que Derrida ¡no era muy argumentativo! (Habermas 1985:228). Para proceder igual que Habermas dejemos sus argumentos a un lado y veamos algunas figuras retóricas que aparecen en su libro. Establece tres puntos clave del texto de Cullers, y por sorpresa los argumentos de Cullers, por tres veces, son de repente los de Derrida (Habermas 1985:229, 231, 232). Sin embargo, hay que reconocer que para Habermas estos argumentos tienen una importancia de segundo rango, así que es casi increíble cuando empieza con el argumento principal de Derrida y otra vez deja a un lado el texto de Derrida y esta vez coge el libro de Mary L. Pratts (Speech Act Theory and Literary Discourse, London, 1977). Toma esta obra por analogía con el argumento de Derrida, lo que no deja de ser chocante al entender que la obra de Pratts defiende a Austin y Searle a los que criticó Derrida (Pålshaugen 1997:75). No carece de comicidad leer este texto de Habermas, pero también deja claro, los rasgos autoritarios del mismo. Pålshaugen concluye el ensayo afirmando que la crítica del lenguaje da al conocimiento una visión dialéctica en la lógica y en la retórica, siendo ambos partes del proceso de conocer.

III. Habermas contra Wittgenstein: ¿hay una ciencia postmoderna?

En Zur Logik der Sozialwissenschaft (Frankfurt a. M.: Suhrkamp Verlag, 1970) Habermas llega a la conclusión de que el lenguaje tiene potencia racio-

6 «Derrida will die Souveränität der Rhetorik über das Gebiet des logischen ausdehnen, um jenes Problem zu lösen, vor dem die totalisierende Vernunftkritik steht". 
nal en virtud de la intersubjetividad (pp. 220-1). Esta conclusión es un producto de su lectura de Wittgenstein. El punto de partida en las ciencias normativas son las reglas intersubjetivas que nosotros como usuarios del lenguaje tenemos que seguir, la gramática de los juegos lingüísticos. Tres son los aspectos de esta filosofía (Pålshaugen 1997:87-8):

1) la idea de que las normas sólo pueden ser criticadas y justificadas a través de una fundamentación discursiva,

2) la idea de que el mejor argumento vence por su propio peso (die gewaltloseren Zwang von Einsicht), y

3) la idea de que la potencia emancipadora de la ciencia está en la (auto)reflexión crítica.

Luego, por ejemplo en Erkenntnis und Interesse (Frankfurt a. M.: Suhrkamp Verlag, 1973), estas investigaciones toman el carácter de programa, es decir, algo que se tiene que defender (para justificar la teoría de la acción comunicativa en el caso de Habermas). Por eso es importante ver qué entiende Habermas por el juego lingüístico y la regla que toma del segundo Wittgenstein.

Los juegos lingüísticos se caracterizan por conectar el lenguaje y la acción (Habermas 1970:238). Entender el lenguaje es conocer algo práctico, no sólo entender símbolos puros sino también comunicar con otros mediante los símbolos. El énfasis está en la dimensión extra-lingüística, el lenguaje como acción social, el uso del lenguaje como interacción social y las relaciones intersubjetivas que se establecen. Este juego no se puede reducir y por lo tanto se establece el «letzte Metasprache» (el último meta-lenguaje). El análisis se concentra en la organización interna de este lenguaje que obviamente consiste en reglas gramaticales (Habermas 1970:240). Está, por así decirlo, analizando el orden interno por el lenguaje mismo que analiza. Se deja ver necesariamente la conexión entre lenguaje y práctica social. El lenguaje no sólo es una lengua sino también una práctica.

El segundo argumento que encuentra Habermas en Wittgenstein trata sobre qué es seguir una regla. El punto clave es que un sujeto por sí sólo no puede seguir una regla, es decir que una regla no puede ser privada. Se refiere al $\$ 202$ (Wittgenstein, Philosophische Untersuchungen, Frankfurt a. M.: Suhrkamp Verlag, 1977), donde Habermas afirma que creer seguir la regla es, 
de hecho, no seguir la regla ${ }^{7}$. Encontramos aquí el elemento constitutivo de la racionalidad comunicativa, o sea, con las reglas tenemos una base desde la que podemos criticar discrepancias. Para los juegos lingüisticos son necesarias dos o más personas y por eso las reglas son necesariamente intersubjetivas. Es una consecuencia directa de la filosofia de Wittgenstein. Habermas va más allá de Wittgenstein y lo dice explícitamente, pero también afirma que si Wittgenstein hubiera desarrollado una teoría de la racionalidad comunicativa sería la misma que defiende Habermas (Pålshaugen 1997:94).

Ahora bien, vayamos al texto de Wittgenstein para ver si Habermas tiene suficiente apoyo para defender su interpretación. En el $\$ 202$ dice: $« Y$ creer que uno sigue la regla es no seguir la reglan ${ }^{8}$. El que Habermas haya añadido "de hecho" no nos interesa tanto como la omisión de la conjunción "y». Ésta se refiere a la primera frase del párrafo: «Por lo tanto seguir la regla es una práctica" ". La palabra "por lo tanto" se refiere al párrafo anterior que está incluido en una serie de párrafos tratando del problema "seguir una regla». La lectura de ambos no apoya la interpretación de Habermas. Veamos por qué.

El tratamiento de «seguir una regla» en Wittgenstein trata de investigar el cómo saber emplear las palabras correctamente. El punto de partida es la expresión del $\$ 197$ : «es como si supiéramos todo el empleo de la palabra de golpe" ${ }^{10}$. El problema es que decimos que entendemos la palabra y a la vez su sentido está en su uso (f́bid.). Entender una frase es entender un lenguaje. Entender un lenguaje es manejar una técnica ( $\$ 199)$. El manejar una técnica parece ser, según Habermas, poder con las reglas del juego. Wittgenstein compara aquí las dos dimensiones con un juego de ajedrez y pregunta si las reglas están ya en mis intenciones o si no sé cómo jugar antes de empezar (\$197). No, responde Wittgenstein, ninguno tiene sentido (Unsinn). La respuesta es, que la conexión entre el juego y las reglas está en la práctica $(\$ 197)$. Hasta cierto punto uno tiene que saber las reglas de antemano, pero no como un saber implícito; se manejan las reglas a través del contacto diario, a través del empleo de las mismas. Esta práctica implica más que las reglas que definen el

7 "Sprachspiel, Intention und Bedeutung. Zu Motiven bei Sellars und Wittgenstein", en WIgGerhauS, R. (Hrsg.) Sprachanalyse und Soziologie, Frankfurt a. M.: Suhrkamp Verlag, 1975:324, "Der Regel zu folgen glauben ist nicht: der Regel (tatsächlich) folgen».

8 "Und der Regel zu folgen glauben ist nicht: der Regel folgen".

9 "Darum ist «der Regel folgen" eine Praxis".

10 "Es ist, als könnten wir die ganze Verwendung des Wortes mit einem Schlag erfassen". 
juego, va más allá de las reglas. La condición mínima es poder interpretar las reglas, la cual en última instancia siempre "puede concordar con la regla» ( $\$$ 198) ${ }^{11}$. Vemos, pues, que Wittgenstein mantiene que la regla es constitutiva en el juego. El problema es que una regla no es absoluta sino que en cada situación concreta mostramos lo que entendemos por seguir la regla y no seguirla (\$201). La interpretación de cada uno está en qué modo realizamos la acción, si cometemos un error podemos intentarlo de nuevo y en estos intentos prácticos está nuestra interpretación de la regla. De la misma manera juzgamos sobre lo dicho, es decir, juzgamos las acciones concretas.

Dejemos que concluya el autor (Pålshaugen 1997:98):

"Ya que las reglas en este sentido [como acciones prácticas] son válidas, existe, como comenta Wittgenstein, una tendencia a decir que cada acción que sigue a una regla es una interpretación... Por esta razón, el haber demostrado que la interpretación de reglas no se puede entender como acto mental donde se calcula la significación de una regla u otra, sino como una acción concreta práctica (p. ej. enunciar una frase) donde el modo concreto de realizar la acción es el que deja ver si uno sigue o no la regla, puede concluir Wittgenstein ahora con el párrafo en el que Habermas encontró el "argumento famoso": seguir la regla es entonces una práctica. Y creer seguir la regla es no seguir la regla».

La diferencia entre Habermas y Wittgenstein se puede resumir así: Habermas mantiene que la intersubjetividad constituye una identidad de sentido para los usuarios del lenguaje, mientras para Wittgenstein la intersubjetividad no está en la interpretación del sentido de la regla ni está implícita en la regla misma sino que está en el hecho de que repetimos el uso, existe un modelo común de aplicaciones que hace de la regla una regla. A la vez, este modelo está condicionado por la interacción entre el uso del lenguaje y la acción práctica que caracterizan la Lebensform de los hombres. Como dice Wittgenstein en el $\$ 122$ a nuestra gramática le falta claridad (Übersehen), lo que es lo contrario de la tesis de Habermas que sostiene que se pueden abarcar las reglas a través de la acción comunicativa de forma universal. Se supone por lo tanto que el lenguaje es objetivo ya que se ha garantizado igualdad entre los participantes del discurso. Habermas produce entonces lo que llama Wittgenstein

11 "Was immer ich tue, ist doch irgendeine Deutung mit der Regel zu vereinbaren». 
(\$109): «Die pneumatische Auffassung des Denkens» (el sin sentido del pensar) y continúa afirmando que no podemos construir una teoría general de la gramática porque siempre se tiene que referir a un contenido/texto concreto.

Concluyo este viaje por los países nórdicos con mi segunda objeción al argumento del autor. A través de los tres ensayos hemos visto como Habermas ha tratado tres autores y que, según Pålshaugen, ha cometido errores graves para su lectura. Ahora bien, supongo que estas tres perspectivas, hasta cierto punto inconmensurables entre sí, representan la opinión del autor. Mi pregunta es entonces si mantener tres puntos de vista inconmensurables es la consecuencia del giro lingǘstico ęcómo pueden estos juicios ser mejores sin que el autor mismo caiga en die gewaltloseren Zwang von Einsicht? Aunque Habermas no ha logrado escapar de la subjetividad de la ilustración ¿̨hay que dejar la búsqueda de lo que hace común nuestra comunicación? Mantener una multitud de contexto ¿ya no presupone que se puede entender o transmitir este sentido, cometiendo, como afirma Habermas, una falacia de expresiones ejecutivas? Aquí cedo la palabra al lector... 